\title{
Cómo fomentar la práctica de ejercicio físico a través de los deberes activos en estudiantes universitarios \\ How to promote exercise among university students through active homework
}

\author{
Aina M Galmes-Panades, Josep Vidal-Conti \\ Universitat de les Illes Balears
}

\begin{abstract}
Resumen. En España el sedentarismo y la inactividad física son elevados en población adulta e infantil, lo que puede influir en las elevadas tasas de sobrepeso y obesidad. El objetivo fue valorar la acogida de los alumnos ante una intervención sobre los hábitos de ejercicio físico a través de los deberes activos y obtener información para el diseño del estudio principal. Así como innovar en estrategias educativas en el ámbito universitario para el fomento y la concienciación de los hábitos saludables. Desde la asignatura de educación para la salud, optativa del grado en educación primaria, especialidad de educación física, se propuso abordar la problemática del sedentarismo y la inactividad física a través de los deberes activos. Los alumnos (n=47) debían realizar un registro de ejercicio físico, cumpliendo con las recomendaciones de la Organización Mundial de la Salud, de manera semanal, a lo largo de toda la asignatura. Para realizar dicho registro los alumnos podían utilizar, en función de la actividad realizada, aplicaciones móviles, pulsómetros o pulseras de actividad, entre otros. Se utilizó la información del registro de los alumnos, así como el foro virtual de la asignatura para la recogida de información. Con los resultados obtenidos en el proyecto piloto se ha diseñado la intervención para el estudio principal, en el que se recogerán diversos parámetros relacionados con el estado de salud, la actividad física y el sedentarismo, con mayor tamaño muestral, diferenciando entre grupo control y grupo intervención. Los deberes activos para la promoción del ejercicio físico han resultado ser un trabajo motivante e innovador, que permite el aprendizaje significativo, positivo para el alumno en el aprendizaje, la salud, y el futuro laboral como maestro. Palabras Clave: Ejercicio físico, deberes activos, educación para la salud, estilo de vida.
\end{abstract}

Abstract. Sedentarism, physical inactivity, and unhealthy eating habits are lifestyle factors that could have an influence on health status. In Spain, sedentarism and physical inactivity are high in both adults and children, which could have a role in the elevated rates of overweight and obesity in these populations. The aim of the present study was to evaluate the effects of an intervention promoting healthy habits to improve students' global health status by means of the methodology of active homework, as well as to work on selfevaluation as a competence. Moreover, to introduce innovative educational strategies promoting and raising awareness on healthy habits at the university level. From the subject of education for health, an elective course within the bachelor program in primary education, specialty of physical education, we attempt to solve the problem of sedentarism and physical inactivity with active homework. Students $(n=47)$ were asked to create a weekly log of their physical exercise throughout the duration of the course, according to the World Health Organization recommendations. To make this log, students could use apps, heart rate monitors, activity wristbands, etcetera. Students' $\log$ information was combined with that obtained from a virtual forum of the course. We designed the intervention for the principal study based on the results from the pilot project, including the collection of health parameters related with physical activity and sedentarism. The intervention was applied to a larger sample, split into control and intervention groups. The active homework represented a motivational and innovative means to promote physical exercise, giving students the chance to acquire significative learnings, which are positive during the teaching-learning process, as well as better health and professional future.

Key words: Physical exercise, Active-homework, health education, lifestyle.

\section{Introducción}

Los efectos positivos del ejercicio físico sobre la salud están ampliamente documentados en todas las edades. Estos efectos incluyen mejoras en la condición física, la salud ósea, la composición corporal, y los biomarcadores cardiometabólicos, todo ello asociado a la prevención de numerosas enfermedades (Vidal-conti, 2016).

La inactividad física es el cuarto factor de riesgo más importante de mortalidad en todo el mundo y se estima que es la causa del 6\% de enfermedades coronarias, el 7\% de diabetes tipo II, 9\% de la mortalidad prematura, el 10\% del cáncer de colon y el 10\% del cáncer de mama (Piercy et al., 2018).

Diferentes organizaciones a nivel mundial han desarrollado guías de práctica de actividad física. La frecuencia, duración e intensidad de la actividad física necesaria para otorgar beneficios en los niños siguen siendo objeto de debate, aunque el consenso general es que los niños y jóvenes

\footnotetext{
Fecha recepción: 11-04-19. Fecha de aceptación: 12-07-19

Aina Maria Galmés Panadés
}

aina.galmes.panades@gmail.com deben acumular un promedio de como mínimo 60 minutos al día de actividad física moderada o vigorosa, con actividades aeróbicas que constituyan la mayor parte de la actividad. En relación a la intensidad del ejercicio, recientes estudios han demostrado que la intensidad vigorosa del ejercicio físico es la más beneficiosa sobre la salud, incluyendo actividades más cortas pero a la vez más intensas, con especiales beneficios sobre parámetros de riesgo cardiovascular (Carson et al., 2014; Hay et al., 2012).

Además del ejercicio físico, se debe tener en cuenta el sedentarismo, ya que las evidencias indican que el sedentarismo prolongado tiene efectos sobre la inflamación relacionada con la obesidad y la diabetes tipo 2 (Allison, Jensky, Marshall, \& Alain, 2013; Galmes-Panades et al., 2019; Healy et al., 2008; Healy, Matthews, Dunstan, Winkler, \& Owen, 2011; Hu, Li, Colditz, Willett, \& Manson, 2003). La diabetes tipo 2 es una de las enfermedades crónicas más prevalentes a nivel mundial, y su origen está muy relacionado con la inactividad física, el sedentarismo y la malnutrición (Chen, Magliano, \& Zimmet, 2012; Falconer et al., 2014), además se relaciona con otras enfermedades crónicas como la obesidad y un elevado riesgo cardio-metabólico. A pesar de ello, todavía hay gran controversia respecto a cuál es la me- 
jor relación actividad física - sedentarismo para prevenir y tratar las enfermedades cardio-metabólicas.

La obesidad es uno de los mayores problemas de los sistemas de sanidad pública del siglo XXI. La acumulación excesiva de grasa se asocia con un incremento del riesgo de enfermedad metabólica, así como enfermedades cardiacas, perdida de independencia, morbilidad y mortalidad (GalmésPanadés, Konieczna, Colom, Morey, \& Romaguera, 2018; Monleón C., Fargueta, M. y Elvira, 2015; Padilla, 2014; Stenholm, Rantanen, Heliövaara, \& Koskinen, 2008).

A pesar de la evidencia científica sobre los beneficios del ejercicio físico, el 80\% de adolescentes no cumple las recomendaciones mínimas de actividad física (Da Cuña Carrera, Lantarón Caeiro, González González, \& Gutiérrez Nieto, 2017; Hallal et al., 2012), que ha descendido en los últimos 20 años (Abarca-Sos, Zaragoza Casterad, Generelo Lanaspa, \& Julián Clemente, 2010), aumentando simultáneamente la prevalencia de sobrepeso y obesidad en niños y adolescentes en las últimas décadas (Marie Ng, Tom Fleming, Margaret Robinson, Blake Thomson, Nicholas Graetz, Christopher Margono, Erin C Mullany et al., 2014).

La adolescencia es el periodo de edad en el que se producen los descensos más pronunciados actividad física (Castillo Viera, Tornero Quiñones, \& García Araujo, 2018; Muntaner-Mas, Vidal-Conti, Cantallops, Borràs, \& Palou, 2017), descendiendo la actividad física entre un 8-10\% cada año en un periodo de 5 años en una muestra de escolares de 12 a 16 años (Bélanger et al., 2009). Además de descender la práctica de ejercicio físico, los niveles de condición física y capacidad aeróbica de los escolares también están descendiendo (Peters et al., 2016). Teniendo en cuenta que el principal indicador de la salud general de los niños es la condición física y en particular la capacidad aeróbica, entendemos que el problema de la inactividad física se ha convertido en un problema de salud pública en España.

Un estudio realizado en 10 ciudades europeas mostró que en adolescentes de entre 12-16 años, los niños practicaban más ejercicio físico y a intensidades más elevadas que las niñas. Al igual que se encontró en otros estudios, en los grupos de edad más jóvenes la práctica de ejercicio físico era mayor, e iba reduciendo según incrementaba la edad (De Cocker et al., 2011). Otro estudio realizado en población española con edades comprendidas entre los 16-18 años obtuvo los mismos resultados (Beltrán-Carrillo, V.J.; Devís-Devís, J. y Peiró-Velert, 2012). Estos resultados son alarmantes, por un lado, por la pérdida de un hábito saludable como la práctica de ejercicio físico, en una etapa fundamental para la adquisición de hábitos que se mantengan a lo largo de la vida adulta (Cavill, Biddle, \& Sallis, 2001). Por otro, por las diferencias entre género, en la que desde una edad muy temprana las mujeres no tienen una buena adherencia a un hábito saludable que tendrá importantes repercusiones sobre su salud a largo plazo.

Un hábito es un mecanismo estable en el tiempo, que crea habilidades o destrezas y puede ser utilizado en varias situaciones de la vida diaria. Un conjunto de hábitos forman las costumbres y formas de comportamiento de las personas ante las diversas situaciones de la vida diaria, por lo que influyen sobre las pautas de conducta a largo plazo y tienen una repercusión sobre el estado de salud, nutrición y bien- estar (Cabrera-Rode, E. Diaz Horta, O. Molina Matos, 2000; Cuadri Fernández, Tornero Quiñones, SierraRobles, \& Padilla Sáez, 2018). Esta repercusión puede ser positiva o negativa; así mismo, los diferentes hábitos de una persona suelen estar interrelacionados, es decir, si una persona tiene un hábito saludable, por ejemplo: realizar ejercicio físico de forma regular, con mayor probabilidad se acompañará de otros hábitos saludables, como no abusar de sustancias nocivas como el alcohol o el tabaco. La adquisición de los hábitos saludables debe realizarse especialmente durante la infancia, y también durante la adolescencia, con el objetivo de que los hábitos que se adquieran desde un primer momento sean saludables (Da Cuña Carrera et al., 2017). Algunos aspectos a tener en cuenta que facilitaran la adquisición de estos hábitos y favorecerán su permanencia a largo plazo son: motivación, comprensión de los beneficios, libertad en la toma de decisiones por parte del niño, así como tener ejemplos a seguir en su entorno cercano, como es la familia y la comunidad educativa (Cabrera-Rode, E. Diaz Horta, O. Molina Matos, 2000).

Los centros escolares representan un lugar idóneo para la promoción de la condición física relacionada con la salud (Heath et al., 2012), y la Educación Física (EF) es la asignatura más indicada para este fin, ya que permite solventar los principales problemas detectados en los adolescentes europeos: la práctica insuficiente de ejercicio físico, la intensidad ligera del ejercicio, y la menor práctica por parte de las niñas, por debajo de las recomendaciones de la OMS. Desde hace años, los adolescentes permanecen muy por debajo del rango de actividad física de intensidad moderada o vigorosa (MVPA) recomendado durante las clases de EF, debiendo ser como mínimo del 50\% (Fröberg, Raustorp, Pagels, Larsson, \& Boldemann, 2017; Hollis et al., 2016), en población española es tan solo es del 10.8\% (Esteban-Cornejo et al., 2016). Siendo conscientes de que la carga lectiva en esta área no puede ser modificada por los docentes, éstos deben introducir cambios en el diseño de sus clases para que supongan un mayor estímulo fisiológico a través del incremento la intensidad de trabajo.

Una nueva metodología para trabajar a intensidad vigorosa en niños y adolescentes de manera saludable es la técnica High Intensity Interval Training (HIIT) o Entrenamiento interválico de alta intensidad. Algunos estudios han evidenciado que el HIIT, en adolescentes, provoca mayores adaptaciones fisiológicas, relacionadas con una reducción del riesgo cardio-metabólico y mejoras en la composición corporal, en comparación con sistemas de entrenamiento tradicionales (Costigan, Eather, Plotnikoff, Taaffe, \& Lubans, 2015; Logan, Harris, Duncan, \& Schofield, 2014). Esta metodología podría contribuir a solucionar el problema de la inactividad física en población infantil y adolescente, aumentando la intensidad de las sesiones de educación física. En los últimos años y en respuesta a esta pandemia, numerosas intervenciones se han centrado en la promoción y evaluación de la actividad física en los centros escolares (Dobbins, DeCorby, Robeson, Husson, \& Tirilis, 2009). Si bien es fundamental el trabajo desde los centros escolares, también lo es la formación de los futuros maestros, para poder actuar correctamente una vez lleguen al centro escolar.

Desde las universidades se debe contribuir a solucionar la problemática del sedentarismo y la inactividad física en la 
infancia y la adolescencia, por un lado, garantizando que los futuros maestros tienen las competencias necesarias para afrontar dicho problema, y, por otro lado, fomentando que los futuros maestros tengan hábitos saludables para poder servir de ejemplo a sus futuros alumnos. De entre los diferentes recursos didácticos, en el presente proyecto se optó por los deberes activos. Los deberes activos consisten en asignar una tarea a los alumnos, que deberán llevar a cabo de forma práctica, y que formara parte de la evaluación de la asignatura. La evaluación universitaria es compleja, ya que debe cumplir, bajo nuestro punto de vista, una función evaluativa, educativa y de aprendizaje. Al abordar los hábitos saludables a través de los deberes activos como metodología innovadora, a través de la autoevaluación basada en evidencias, se favorecer el autoconocimiento, el espíritu crítico y la autonomía del alumno (Ángel Pérez Pueyo, Belén Tabernero Sánchez, Víctor M.López Pastor, Nuria Ureña Ortín, Encarna Ruiz Lara, Marta Caplloch Bujosa, 2008). Además, se garantiza que los alumnos cumplen las recomendaciones de ejercicio físico en relación con la salud.

En el marco universitario europeo actual, el proceso de enseñanza-aprendizaje se basa en las competencias. Una competencia es un saber hacer complejo, fruto de la adecuación e integración de conocimientos, habilidades y actitudes, utilizados eficazmente en cada situación (Villardón-Gallego, 2006). La educación basada en competencias tiene una estrecha relación con la innovación en la evaluación. Por un lado, se deben evaluar conocimientos, habilidades y actitudes; y por otro, la situación a evaluar debe ser una situación muy similar a una situación real fuera del contexto educativo (Villardón-Gallego, 2006).

Las competencias básicas son aquellas competencias transferibles a otros contextos diferentes al educativo, como la evaluación (Bolívar, 2018) y muy relacionado con esta, la capacidad crítica y la reflexión.

De acuerdo con un estudio realizado en 10 universidades públicas españolas, los estudiantes universitarios no se sienten competentes en lo que a la evaluación y al aprendizaje autónomo se refiere (Rodríguez Gómez, Gregorio; Ibarra Saiz, María Soledad; Cubero Ibáñez, 2018). Para una adecuada adaptación en el mundo laboral es importante que los estudiantes universitarios sean competentes evaluando, ya que, en la mayoría de los escenarios profesionales, deberán evaluar a personas, situaciones o proyectos. En el caso de futuros maestros esta competencia adquiere mayor importancia, ya que además de evaluar a sus alumnos, deberán evaluar sus programaciones para garantizar una enseñanza de calidad.

Entendemos por autoevaluación, el proceso que un sujeto pone en práctica al valorar sus actuaciones y producciones (Rodríguez Gómez, Gregorio; Ibarra Saiz, María Soledad; Cubero Ibáñez, 2018), potenciando el desarrollo de competencias a través de la reflexión (Villardón-Gallego, 2006).

La autoevaluación y la reflexión son dos procesos inseparables. La autorreflexión es fundamental para el aprendizaje, ya que favorece la toma de conciencia de las propias estrategias, barreras y facilidades en este proceso (VillardónGallego, 2006). La autoevaluación es una competencia fundamental en cualquier profesión, para poder analizar y mejorar el propio trabajo.
De entre diversas propuestas sobre cómo mejorar la evaluación (Boud, 2010; Gómez-Ruiz \& Rodríguez Gómez, Gregorio; Ibarra Sáiz, 2013), a través de la autoevaluación se trabajan las siguientes: Involucrar a los estudiantes en su aprendizaje, utilizar la retroalimentación para mejorar el aprendizaje, compartir la responsabilidad entre docentes y estudiantes e iniciar a los estudiantes en la evaluación.

En definitiva, basándonos en una amplia revisión bibliográfica, se considera que son necesarias actuaciones para, por un lado, aumentar la práctica de ejercicio físico en todos los grupos poblacionales, centrándonos en el presente estudio en adultos jóvenes; Y, por otro lado, innovar en docencia universitaria para lograr aprendizajes significativos y formar a alumnos competentes como maestros y como ciudadanos.

De acuerdo con lo expuesto anteriormente, el objetivo del presente proyecto es valorar la acogida de los alumnos ante una intervención sobre los hábitos de ejercicio físico a través de los deberes activos y obtener información para el diseño del estudio principal.

\section{Material y métodos}

Dentro del marco de la asignatura optativa de educación para la salud, en el grado de educación primaria, mención de educación física, se realizó un proyecto piloto, para analizar la acogida de los alumnos a este tipo de proyectos, y sentar las bases para un futuro proyecto, con mayor tamaño muestral.

\section{Participantes}

La muestra estuvo constituida por 47 alumnos de cuarto curso de la Universitat de les Illes Balears. Los alumnos tenían edades comprendidas entre los 22 y los 35 años, y el grupo estaba compuesto por 20 mujeres (42.5\%) y 27 hombres (57.5\%). Todos los estudiantes tenían nacionalidad española y residían en Mallorca, España. Como criterio de inclusión los alumnos debían estar matriculados en la asignatura de Educación para la salud. Como criterio de exclusión los alumnos no podían presentar contraindicación médica para la práctica de ejercicio físico.

\section{Recogida de información}

Para la realización de este estudio se informó previamente a los participantes de las características del mismo y su relación con la asignatura.

La información se obtuvo a partir del diario de los alumnos, qué consistía en realizar un registro de evidencias sobre el cumplimiento de las recomendaciones de la OMS (World Health Organization, 2010), realizando 60 minutos de ejercicio físico, 5 días a la semana. La forma de evidenciar dicho cumplimiento era libre, podían utilizar aplicaciones móviles, pulseras de actividad, fotografías, mapas, etc. Y debían realizar una autoevaluación sobre si habían cumplido con las recomendaciones, y si habían sido capaces de evidenciarlo correctamente. De este modo, con los deberes activos se trabajó la indagación, el aprendizaje autónomo, el espíritu crítico y la reflexión. El registro de ejercicio físico debía llevarse a cabo durante 6 semanas consecutivas, y disponían de una semana adicional para realizar la evaluación. 
$\mathrm{Al}$ establecer cada alumno sus criterios de evaluación, con la única premisa de que debían haber superado los 60 minutos/5 días a la semana, las evaluaciones fueron muy personalizadas y diversas. Algunos alumnos valoraron más el esfuerzo que habían realizado, otros las mejoras que habían logrado a lo largo de la tarea, y otros mantuvieron sus rutinas de ejercicio, sin implicar la tarea un esfuerzo adicional o una mejora en la condición física, pero valoraron que cumplían las recomendaciones. Otra parte del registro era una reflexión sobre el proceso y el resultado a nivel personal, así como su opinión sobre el trabajo, con lo que se obtuvo información cualitativa sobre todo el proceso. Como parte final de la tarea, los alumnos individualmente explicaron su trabajo, dando un ejemplo de evidencia, explicando los criterios de evaluación utilizados y comentando su reflexión.

Antes de iniciar el registro se explicó a los alumnos que información debían registrar, dónde podían encontrar las recomendaciones de la OMS, y una lista de aplicaciones y productos que podían utilizar para registrar su ejercicio físico. Además, se habilito un foro a través del aula virtual en el que los alumnos podían proponer nuevas app’s para el registro, y comentar sus experiencias con las formas de registro que utilizaban, tales como podómetro, pulsómetro, pulsera de actividad, app's o fotografías entre otros.

La asignatura se centraba en cómo la educación física (EF) puede contribuir a la salud global de los alumnos directamente, y de la población general indirectamente. Entre otros contenidos se trabajaron la alimentación, el ejercicio físico, o la educación postural. Por lo que, dentro de las genéricas recomendaciones, los alumnos podían adecuar el ejercicio físico a sus gustos y necesidades, basándose en los conocimientos adquiridos a lo lago de la asignatura, para llevar a cabo un hábito saludable. Algunas adecuaciones podían ser: combinar ejercicio aeróbico con ejercicios de fuerza y flexibilidad, realizar la mayor parte del ejercicio a intensidad moderada o vigorosa, seguir una progresión en aumento al empezar una rutina, o variar el tipo de ejercicio, duración o intensidad para mejorar la condición física. Estas adaptaciones las podrían tener en cuenta en su autoevaluación. Por ejemplo, podrían valorar positivamente el haber combinado ejercicio aeróbico con ejercicios de fuerza, o realizar ejercicio 6 días a la semana en lugar de 5 días.

Dado que el objetivo de presente estudio piloto era valorar la acogida de los alumnos ante una intervención de estas características y ayudar al diseño del estudio principal, los análisis realizados han sido descriptivos, expresando en porcentajes la cantidad de alumnos que han hecho referencia a aspectos concretos sobre los beneficios y características de la intervención.

\section{Resultados}

La muestra total en el estudio fue de 47 alumnos, de los cuales 16 hicieron referencia a los siguientes aspectos en sus reflexiones: mejoras en la salud referidas a pérdida de peso y/o deshabituación tabáquica, mejora de la condición física, beneficios sociales del ejercicio, intención de continuar con las recomendaciones de la OMS a largo plazo, el carácter motivador del trabajo, o la concienciación que habían adquirido sobre el ejercicio físico que realizaban habi-
Tabla 1.

Valoración de la influencia de los deberes activos sobre los alumnos, a través de las reflexiones personales.

\begin{tabular}{lcc}
\hline \multicolumn{1}{c}{ Ítem } & Número alumnos (n=16) & Porcentaje \\
\hline $\begin{array}{l}\text { Mejoras de la salud referidas a pérdida de peso y/o } \\
\text { deshabituación tabáquica }\end{array}$ & 9 & $56.6 \%$ \\
\hline Mejora de la condición física & 12 & $75 \%$ \\
\hline Beneficios sociales del ejercicio & 7 & $43.8 \%$ \\
\hline $\begin{array}{l}\text { Intención de continuar con las recomendaciones de la } \\
\text { OMS a largo plazo }\end{array}$ & 9 & $56.6 \%$ \\
\hline Carácter motivador del trabajo & 10 & $62.5 \%$ \\
\hline Concienciación sobre los hábitos de ejercicio físico & 11 & $68.8 \%$ \\
\hline
\end{tabular}

Concienciación sobre los hábitos de ejercicio físico

\begin{tabular}{|c|c|}
\hline $\begin{array}{c}\text { Estudio Piloto, } \mathrm{n}=47 \\
\text { Febrero 2018-Mayo } 2018\end{array} \longrightarrow \begin{array}{c}\text { Estudio principal } \\
\text { Diseño: Diciembre 2017 - Enero } 2019\end{array}$ \\
\hline
\end{tabular}

\begin{tabular}{|c|c|c|c|}
\hline \multicolumn{2}{|c|}{$\begin{array}{c}\text { Estudio principal } \\
\text { Análisis y publicación de resultados: } \\
2021 \\
\end{array}$} & \multicolumn{2}{|c|}{\begin{tabular}{|c|} 
Estudio principal \\
Puesta en práctica: Mayo 2019 - Setiembre \\
2020 \\
\end{tabular}} \\
\hline \multicolumn{4}{|c|}{ Figura 1. Línea temporal del estudio } \\
\hline \begin{tabular}{l}
\multicolumn{1}{c}{ Pretest } \\
-4 meses antes de \\
iniciar la intervención \\
Mayo 2019 \\
-A nivel basal \\
Setiembre 2019 \\
En la Universidad: \\
-Consentimiento \\
informado \\
-Test físicos \\
-Cuestionarios auto- \\
reportados \\
-Acelerómetro \\
-Datos \\
antropométricos - \\
Composición corporal \\
determinada con \\
bioimpedancia - \\
Tensión arterial
\end{tabular} & $\begin{array}{l}\text { Se le ofrecerá } \\
\text { en los test físi } \\
\text { antropométric } \\
\text { corporal. Se l } \\
\text { de seguir uno } \\
\text { relacionados } \\
\text { ejercicio físic } \\
\text { informativo e }\end{array}$ & $\begin{array}{l}\text { rupo control } \\
\text { la información obtenida } \\
\text { cos, las medidas } \\
\text { as y la composición } \\
\text { e recordara la importancia } \\
\text { s hábitos saludables } \\
\text { con la alimentación y el } \\
\text { o a través material } \\
\text { n el aula virtual. }\end{array}$ & \begin{tabular}{|l|}
\multicolumn{1}{|c|}{ Postest } \\
-Al finalizar la \\
intervención \\
Diciembre 2019 \\
-Al año de haber \\
finalizado la \\
intervención \\
Setiembre 2020 \\
En la Universidad: \\
-Test físicos \\
-Cuestionarios auto- \\
reportados \\
-Acelerómetro \\
-Datos \\
antropométricos - \\
Composición corporal \\
determinada con \\
bioimpedancia - \\
Tensión arterial \\
\end{tabular} \\
\hline
\end{tabular}

Figura 2. Diagrama de flujo del proceso

Tabla 2.

Variables propuestas para el estudio principal

\begin{tabular}{|c|c|c|c|}
\hline Nom & nbre & Unidad de medida & Objeto de medida \\
\hline \multirow{3}{*}{ Batería ALPHA } & Course-navette & Numero de periodos & $\begin{array}{l}\text { Capacidad aeróbica - } \\
\text { Condición física }\end{array}$ \\
\hline & Dinamómetro manual & $\begin{array}{l}\text { Fuerza máxima en } \\
\text { kilogramos }\end{array}$ & $\begin{array}{l}\text { Fuerza en miembros } \\
\text { superiores - Condición } \\
\text { física }\end{array}$ \\
\hline & Test de agilidad 10X5 & Tiempo en segundos & $\begin{array}{l}\text { Velocidad - Condición } \\
\text { física }\end{array}$ \\
\hline $\begin{array}{l}\text { Composición corporal } \\
\text { con bioimpedancia }\end{array}$ & & $\begin{array}{l}\text {-Peso, kg } \\
\text {-Masa magra, kg } \\
\text {-Masa ósea, } \mathrm{kg} \\
\text {-Masa grasa, } \mathrm{kg} \\
\text {-Masa grasa, \% } \\
\text {-Agua, \% } \\
\text {-Grasa visceral, rango }\end{array}$ & $\begin{array}{l}\text { Composición corporal - } \\
\text { detectar riesgos } \\
\text { asociados a } \\
\text { enfermedades } \\
\text { cardiometabólicas }\end{array}$ \\
\hline Datos antropométricos & & $\begin{array}{l}\text {-Talla } \\
\text {-IMC } \\
\text {-Perímetro de cintura, cm } \\
\text {-Perímetro de cadera, } \mathrm{cm} \\
\text {-Perímetro de cuello, } \mathrm{cm} \\
\text {-Índice cintura-cadera }\end{array}$ & $\begin{array}{l}\text { Información } \\
\text { antropométrica - detectar } \\
\text { riesgos asociados a } \\
\text { enfermedades } \\
\text { cardiometabólicas } \\
\end{array}$ \\
\hline $\begin{array}{l}\text { Tensión arterial } \\
\text { sistólica y diastólica }\end{array}$ & & $\mathrm{mmHg}$ & $\begin{array}{l}\text { Tensión arterial - } \\
\text { determinar posibles } \\
\text { asociaciones entre } \\
\text { variables de exposición } \\
\text { y de resultado }\end{array}$ \\
\hline Cuestionario STEPS & & Puntos & $\begin{array}{l}\text { Hábitos tóxicos y nivel } \\
\text { socioeconómico }\end{array}$ \\
\hline Cuestionario SF-36 & & Puntos & Nivel de bienestar \\
\hline $\begin{array}{l}\text { Cuestionario de } \\
\text { frecuencia de } \\
\text { consumo de alimentos } \\
\end{array}$ & & $\begin{array}{l}\text { Veces de consumo por } \\
\text { unidad de tiempo }\end{array}$ & Tipo de alimentación \\
\hline Cuestionario GPAQ & & $\begin{array}{l}\text { Frecuencia de práctica y } \\
\text { tipo de actividad }\end{array}$ & $\begin{array}{l}\text { Hábitos de ejercicio } \\
\text { físico }\end{array}$ \\
\hline Cuestionario NHS & & Horas de sedentarismo & Hábitos de sedentarismo \\
\hline Acelerómetro & & $\begin{array}{l}\text { Aceleración: tiempo, } \\
\text { intensidad y tipo de } \\
\text { actividad realizada }\end{array}$ & $\begin{array}{l}\text { Hábitos de ejercicio } \\
\text { físico }\end{array}$ \\
\hline $\begin{array}{l}\text { Foro de participación } \\
\text { voluntaria }\end{array}$ & & $\begin{array}{l}\text { Número y tipo de } \\
\text { intervención }\end{array}$ & Motivación \\
\hline Excursión en grupo & & $\begin{array}{l}\text { Duración, intensidad, } \\
\text { numero de excursiones }\end{array}$ & $\begin{array}{l}\text { Aspecto social de la } \\
\text { práctica de ejercicio } \\
\text { físico }\end{array}$ \\
\hline
\end{tabular}

tualmente y los cambios que consideraban que debían hacer (Tabla 1). Los aspectos a los que se hizo más referencia son: la mejora de la condición física, en el 75\% de las ocasiones, la concienciación sobre los hábitos de ejercicio, en el 68.8\% de las ocasiones, y el carácter motivador del trabajo, en el 62.5\% de las ocasiones.

Los alumnos participación en el foro del aula virtual que 
se utilizó como una herramienta para la resolución de dudas, y espacio para hacer sugerencias. Además, fue una herramienta para el profesorado, para detectar el grado de motivación de los alumnos, ya que la participación en el foro, además de ser voluntaria, no afectaba a la nota de la asignatura. Se registraron 11 entradas al foro, todas ellas relacionadas con la aportación de diferentes aplicaciones móviles para el registro de AF, además de estrategias y comentarios que incentivaban a los demás participantes a la realización del registro diario.

\section{Discusión}

Con la intervención llevada a cabo, se pretendían detectar las necesidades relacionadas con la práctica de ejercicio físico en el ámbito universitario, así como las características del entorno para garantizar la adecuación del proyecto. Además, el objetivo a corto plazo fue promover los hábitos saludables para mejorar la salud global del alumnado, a través de los deberes activos, fomentando la autoevaluación como competencia.

A partir de la información obtenida a través del estudio piloto, se ha diseñado un proyecto más ambicioso, con el objetivo de analizar el efecto de una intervención de actividad física a través de deberes activos en una muestra de estudiantes universitarios sobre la salud global de los estudiantes.

A continuación, se presenta la línea temporal de los dos estudios.

En el proyecto que se llevará a cabo, se incluirá a los alumnos de tercer curso como grupo intervención, y a los alumnos de cuarto curso como grupo control, ambos cursos de la especialidad de educación física, llegando a tener una muestra aproximada de $n=100$.

El análisis estadístico estará formado, por una parte, por la descripción de los datos utilizando los test estadísticos de análisis de varianza (anova) de un factor y Chi cuadrado $\left(\mathrm{X}^{2}\right)$; son técnicas que se pueden usar para comparar medias de dos o más variables. Se usa el test de análisis de varianza de un factor para variables continuas y el test Chi cuadrado para variables categóricas. Por otro lado, los efectos de la intervención sobre las diferentes variables se analizarán mediante análisis de regresión lineal multi-ajustados, y ecuaciones de estimación generalizada (GEE) a través de medidas repetidas. Todos los análisis se controlarán estadísticamente por las siguientes variables de confusión: sexo, edad y grupo de intervención.

Gracias a la información recogida a través de las reflexiones de los alumnos, se plantean varias incorporaciones para el futuro proyecto, destinadas a mejorar la intervención y la recogida de resultados, para garantizar un adecuado análisis de los efectos de la intervención.

Todas las pruebas y cuestionarios que se presentan a continuación se realizarán en cuatro ocasiones: Cuatro meses antes de iniciar la intervención, a nivel basal, al finalizar la intervención, y al año de haber finalizado la intervención.

En relación a las mejoras de salud referidas a pérdida de peso y/o deshabituación tabáquica, se registrara la siguiente información: La condición física, se medirá a través de la batería ALPHA (Castillo Garzón, 2009), compuesta por los test físicos de la course-navette (García \& Secchi, 2014), el dinamómetro manual y el test de agilidad $10 \times 5$, todos ellos test validados, que permitirán obtener información sobre la condición física global de los alumnos. Todos los test se realizarán en las instalaciones de la universidad, por personal cualificado y de forma estandarizada.

La composición corporal se realizará a través de bioimpedancias, permitiendo diferenciar los compartimentos de: masa grasa, masa ósea y masa magra, así como la grasa visceral. Además, también se tendrán en cuenta datos antropométricos como son el perímetro de cintura, cadera y cuello, o la altura, y algunos índices derivados como el índice de masa corporal (IMC) o en índice cintura-cadera. Estas mediciones se realizarán en las instalaciones de la universidad, por personal cualificado y de forma estandarizada.

La tensión arterial se medirá de forma estandarizada, con el alumno en posición sedente, realizando 3 tomas en cada brazo, dejando 5 minutos entre mediciones. Se registrarán las 3 mediciones de cada brazo, y se calculara la media de las 3 mediciones.

Para conocer los hábitos tóxicos y los datos socioeducativos de los alumnos se utilizará el cuestionario STEPS (Organización mundial de la salud(OMS), 2005). Para conocer su nivel de bienestar se utilizará el cuestionario SF36. Se utilizará un cuestionario de frecuencia de consumo de alimentos validado en población española (Fernández-Ballart et al., 2010) para conocer si el cambio sobre los hábitos de ejercicio se acompaña de otros hábitos saludables como la alimentación. El test Global Physical Activity Questionnaire (GPAQ) o cuestionario global de actividad física (Organización mundial de la salud (OMS), 2012) se utilizará para conocer los hábitos de ejercicio físico de los alumnos antes y después de la intervención, y pasado un año de la intervención. El GPAQ es un cuestionario validado para su utilización en población entre 16 y 65 años (Ara et al., 2015). Durante la última década son numerosos los estudios que apuntan al sedentarismo como un factor determinante en la salud cardio-metabólica, teniendo igual o más importancia que el ejercicio físico (Cooper et al., 2012; Ekelund et al., 2016; Falconer et al., 2014; Healy et al., 2007; Wilmot et al., 2012). Por lo que en el futuro estudio se medirá el tiempo total de sedentarismo de acuerdo con el cuestionario validado Nurses’ Health Study questionnaire (NHS) (MartínezGonzález, López-Fontana, Varo, Sánchez-Villegas, \& Martinez, 2005). El sedentarismo se define como toda actividad que requiera un coste $d » 1.5$ MET como permanecer en posición de sentado o tumbado (Tremblay, 2012). El cuestionario consiste en una recopilación de preguntas abiertas sobre el tiempo diario dedicado en el último año a mirar la televisión, estar sentado usando el ordenador, estar sentado realizando actividades de ocio, trabajo, conducir, viajar en algun tipo de transporte sentado, etc. y tiempo total sentado. Consideramos fundamental recoger informacion tanto de hábitos de actividad física como de sedentarismo, entendiendo que una persona puede cumplir las recomendaciones de ejercicio físico en tiempo e intensidad, y al mismo tiempo acumular un tiempo sedentario superior al recomendado.

Dada la importancia del ejercicio físico y el sedentarismo, además de recoger dicha información con cuestionarios validados, se recogerá con accelerometros. Los alumnos lleva- 
rán un acelerómetro ActiGraph durante 7 días consecutivos, 24 horas al día, en la mano no dominante. Es un instrumento que recoge datos sobre aceleración y proporciona información objetiva sobre la intensidad de los movimientos corporales, puediendo identificar la intensidad del ejercicio realizado o la falta de movimiento (Matthews et al., 2008). Al recogerse las aceleraciones en relacion a las horas del día, se pueden identificar momentos más activos o más sedentarios de forma precisa (Healy et al., 2011). Como variable estimativa de la motivación en el proyecto por parte de los alumnos, se utilizará el grado de participación en el foro habilitado para hacer comentarios sobre el registro de ejercicio físico.

Por lo que hace referencia a los beneficios sociales del ejercicio, será obligatoria realizar una excursión en grupo, que deberá acreditarse con una foto del grupo durante la excursión. Además, se valorará positivamente el desnivel de la excursión, por los beneficios del ejercicio físico vigoroso sobre la salud.

Respecto a la intención de continuar con las recomendaciones de la OMS a largo plazo, se realizará un seguimiento al año de la intervención, para analizar si los niveles de ejercicio físico se han mantenido.

Numerosos estudios apuntan a que, cumpliendo con las recomendaciones de la OMS, aumenta la salud, mejora la condición física y reduce el riesgo de morbilidad. Con el futuro estudio, además de confirmar dichas afirmaciones, se podrán analizar relaciones de dosis-respuesta de ejerciciosedentarismo con niveles de condición física y parámetros de salud, así como llevar a la práctica metodologías novedosas en el campo de la docencia universitaria relacionadas con la salud.

A pesar de ser necesario el aumento de la intensidad en las sesiones de educación física y garantizar un mayor tiempo de compromiso motriz, las medidas para garantizar los hábitos saludables en la población infantil y adolescente deben ir más allá. Si bien es fundamental el incremento de horas semanales de educación física, hay muchos factores que influyen, más allá de la voluntad del maestro de EF, como puede ser: el Decreto de cada Comunidad Autónoma, el claustro de profesorado del centro, o la disponibilidad de espacios y horas de EF. Por esta razón proponemos la utilización de los deberes activos en la educación primaria y secundaria, como herramienta para garantizar el cumplimiento de las recomendaciones de la OMS sobre la práctica de ejercicio físico.

Los proyectos expuestos abordan campos muy diversos y al mismo tiempo muy interrelacionados. Por un lado, la investigación universitaria y la innovación educativa, por otro lado, la promoción de los hábitos saludables, y como tercer pilar, el aprendizaje significativo y la translación de estos aprendizajes al ámbito laboral por parte de los alumnos.

En la educación universitaria se han llevado a cabo algunas experiencias docentes en las que se hace partícipe al alumno del proceso de evaluación (Rodríguez-Gómez, IbarraSáiz, \& Gómez-Ruiz, 2010), pero todavía es una práctica menos habitual de lo que cabría esperar, dados los aprendizajes y competencias que se le atribuyen. En 1999 un total de 19 países europeos firmaron un tratado, que tenía como objetivo el establecimiento para 2010 de un Espacio Europeo de
Educación Superior (EEES) que esperaba facilitar la empleabilidad, la movilidad y el reconocimiento de los títulos universitarios en Europa. Comúnmente este acuerdo se conoce como Plan Bolonia. Con el plan Bolonia se introdujo un cambio significativo en el modelo educativo, adquiriendo una gran importancia el aprendizaje por competencias y la participación activa del alumno en su propio aprendizaje. En el presente proyecto, la realización del diario ya es en sí una actividad formativa y reflexiva. Pero al ir más allá, promoviendo la autoevaluación trabajamos la competencia de la evaluación, imprescindible para todos los graduados, y especialmente para un maestro de educación primaria.

Los hábitos son difíciles de adquirir y mantener, suponen un esfuerzo, requieren cambios en las rutinas personales, y pueden empujar a las personas fuera de su zona de confort. A través del presente proyecto, con los deberes activos se ha eliminado el principal problema de las intervenciones que promueven realizar un cambio de hábitos, por las dificultades relacionadas con la fuerza de voluntad o de interés. Al ser una parte evaluable de la asignatura se convirtió en una tarea obligatoria. Gracias a este carácter obligatorio se consiguió que la totalidad de los alumnos siguieran las recomendaciones de ejercicio físico saludable, un hecho muy poco frecuente en estudios de estas características, donde el cumplimiento de la intervención está en manos del participante, y por lo tanto es muy difícil de controlar.

La principal debilidad del presente estudio es que es un estudio piloto, realizado a una muestra reducida de participantes.

Como fortalezas del presente estudio, destacar la innovación en su planteamiento, por el hecho de utilizar los deberes activos como metodología innovadora para la promoción de la salud, y la utilización efectiva de la autoevaluación en docencia universitaria. Gracias a estos resultados se realizarán un número elevado de pruebas objetivas y validadas, así como cuestionarios validados. Además, el estudio contará con un grupo control y un grupo intervención, lo que permitirá controlar los efectos de la intervención. Todo ello facilitara que los resultados que se obtengan sean de interés científico y con gran aplicabilidad en el ámbito de la docencia. Con ello se esperan aportar nuevos recursos metodológicos para fomentar el incremento de práctica de AF en la docencia universitaria.

\section{Conclusiones}

El presente trabajo describe las necesidades, así como las bases y aspectos metodológicos de una intervención para la promoción de la salud a través de los deberes activos en la docencia universitaria, en alumnos del grado de educación primaria, de la especialidad de educación física, a través de metodologías innovadoras, que favorecen el aprendizaje significativo.

El proyecto piloto tuvo una buena aceptación entre el alumnado, y su cumplimiento fue del 100\%. La hipótesis es que, a través de los deberes activos como método de aprendizaje significativo, se mejorara la salud global de los alumnos, y su concienciación sobre la importancia de los hábitos saludables, manteniendo estos hábitos a largo plazo y, en consecuencia, un buen estado de salud. 
Así mismo, al tratarse de futuros maestros es fundamental el aspecto metodológico del proyecto, con lo que se espera animar a los alumnos y dotarlos de las herramientas necesarias para que puedan poner en práctica proyectos similares en su futura vida profesional, como puede ser llevar a la práctica los deberes activos en educación primaria.

\section{Referencias}

Abarca-Sos, A., Zaragoza Casterad, J., Generelo Lanaspa, E., \& Julián Clemente, J. A. (2010). Comportamientos sedentarios y patrones de actividad física en adolescentes. Revista Internacional de Medicina y Ciencias de La Actividad Fisica y Del Deporte, 10(39), 410-427.

Allison, M.A., Jensky, N. E., Marshall, S. J., \&Alain, G. (2013). Sedentary Behavior and Adiposity-Associated Inflammation The Multi-Ethnic Study of Atherosclerosis, 42(1), 8-13. https://doi.org/10.1016/ j.amepre.2011.09.023.Sedentary

Ángel Pérez Pueyo, Belén Tabernero Sánchez, Víctor M.López Pastor, Nuria Ureña Ortín, Encarna Ruiz Lara, Marta Caplloch Bujosa, N. G. F. y F. J. C. O. (2008). Evaluación formativa y compartida en la docencia universitaria y el Espacio Europeo de Educación Superior: cuestiones clave para su puesta en práctica. Revista de Educación, 347(435-451), 435-451.

Ara, I., Aparicio-Ugarriza, R., Morales-Barco, D., Aznar, S., Nascimento De Souza, W., Mata, E., \& González-Gross, M. (2015). Evaluación de la actividad física en la población general; cuestionarios validados. Rev Esp Nutr Comunitaria, 21, 209-214. https://doi.org/10.14642/ RENC.2015.21.sup1.5067

Bélanger, M., Gray-Donald, K., O’Loughlin, J., Paradis, G., Hutcheon, J., Maximova, K., \& Hanley, J. (2009). Participation in organised sports does not slow declines in physical activity during adolescence. International Journal of Behavioral Nutrition and Physical Activity, 6, 1-6. https://doi.org/10.1186/1479-5868-6-22

Beltrán-Carrillo, V.J.; Devís-Devís, J. y Peiró-Velert, C. (2012). Original Physical Activity and Sedentary Behaviour in. Revista Internacional de Medicina y Ciencias de La Actividad Física y El Deporte, 12, 123-137. https:// doi.org/10.1177/0886260513505143

Bolívar, A. (2018). El discurso de las competencias en España: educación básica y educación superior. REDU: Revista de Docencia Universitaria, ISSN 1887-4592, Vol. 16, $N^{\circ} .1,2018,16(1), 23$.

Boud, D. (2010). Evaluacion 2020. Siete propuestas para la reforma de la evalaucion en la educacion superior. Evaluacion En Contextos Formativos, 1-11. https:// doi.org/10.1037/e556822011-001

Cabrera-Rode, E. Diaz Horta, O. Molina Matos, G. (2000). Formación de hábitos alimentarios y de estilos de vida sal udabl es. Curriculo de Educación Inicial, 331-366.

Carson, V., Rinaldi, R. L., Torrance, B., Maximova, K., Ball, G. D. C., Majumdar, S. R., ... McGavock, J. (2014). Vigorous physical activity and longitudinal associations with cardiometabolic risk factors in youth. International Journal of Obesity, 38(1), 16-21. https://doi.org/10.1038/ ijo.2013.135
Castillo Garzón, M. J. (2009). The Alpha Health-Related Fitness Test Battery For Children and Adolescents, 134.

Castillo Viera, E., Tornero Quiñones, I., \& García Araujo, A. J. (2018). Relación entre actividad física , alimentación y familia en edad escolar Relationship between physical activity, nutrition , and family in school-aged youth. Retos, 34(Imc), 85-88.

Cavill, N., Biddle, S., \& Sallis, J. F. (2001). Health Enhancing Physical Activity for Young People: Statement of the United Kingdom Expert Consensus Conference. Pediatric Exercise Science, 13(1), 12-25. https://doi.org/ 10.1123/pes.13.1.12

Chen, L., Magliano, D. J., \& Zimmet, P. Z. (2012). The worldwide epidemiology of type 2 diabetes mellitus Present and future perspectives. Nature Reviews Endocrinology, 8(4), 228-236. https://doi.org/10.1038/ nrendo.2011.183

Cooper, A. R., Sebire, S., Montgomery, A. A., Peters, T. J., Sharp, D. J., Jackson, N., ... Andrews, R. C. (2012). Sedentary time, breaks in sedentary time and metabolic variables in people with newly diagnosed type 2 diabetes. Diabetologia, 55(3), 589-599. https://doi.org/10.1007/ s00125-011-2408-X

Costigan, S. A., Eather, N., Plotnikoff, R. C., Taaffe, D. R., \& Lubans, D. R. (2015). High-intensity interval training for improving health-related fitness in adolescents: A systematic review and meta-analysis. British Journal of Sports Medicine, 49(19), 1253-1261. https://doi.org/ 10.1136/bjsports-2014-094490

Cuadri Fernández, J., Tornero Quiñones, I., SierraRobles, Á., \& Padilla Sáez, J. M. (2018). Revisión sistemática sobre los estudios de intervención de actividad física para el tratamiento de la obesidad Systematic Review of Physical Activity Programs for the treatment of Obesity, 33, 261266.

Da Cuña Carrera, I., Lantarón Caeiro, E. M., González González, Y., \& Gutiérrez Nieto, M. (2017). REPERCUSIÓN DEL SEDENTARISMO EN LA RESPUESTA CARDIORRESPIRATORIAEN ESTUDIANTES UNIVERSITARIOS. Rev.Int.Med.Cienc.Act.Fís.Deporte, 17, 367-378. https://doi.org/https://doi.org/10.15366/ rimcafd2017.66.010

De Cocker, K., Ottevaere, C., Sjöström, M., Maöreno, L.A., Wrnberg, J., Valtueña, J., ... De Bourdeaudhuij, I. (2011). Self-reported physical activity in European adolescents: Results from the HELENA (Healthy Lifestyle in Europe by Nutrition in Adolescence) study. Public Health Nutrition, 14(2), 246-254. https://doi.org/10.1017/ S1368980010000558

Dobbins, M., DeCorby, K., Robeson, P., Husson, H., \& Tirilis, D. (2009). Cochrane review: School-based physical activity programs for promoting physical activity and fitness in children and adolescents aged 6-18. EvidenceBased Child Health: A Cochrane Review Journal, 4(4), 1452-1561. https://doi.org/10.1002/ebch.461

Ekelund, U., Steene-Johannessen, J., Brown, W. J., Fagerland, M. W., Owen, N., Powell, K. E., ... Lee, I. M. (2016). Does physical activity attenuate, or even eliminate, the detrimental association of sitting time with mortality? A 
harmonised meta-analysis of data from more than 1 million men and women. The Lancet, 388(10051), 1302-1310. https://doi.org/10.1016/S0140-6736(16)30370-1

Esteban-Cornejo, I., Martinez-Gomez, D., Garcia-Cervantes, L., Ortega, F. B., Delgado-Alfonso, A., Castro-Piñero, J., \& Veiga, O. L. (2016). Objectively Measured Physical Activity During Physical Education and School Recess and Their Associations With Academic Performance in Youth: The UP\&amp;DOWN Study. Journal of Physical Activity and Health, 1-24. https://doi.org/10.1123/ jpah.2016-0192

Falconer, C. L., Cooper, A. R., Walhin, J. P., Thompson, D., Page, A. S., Peters, T. J., ... Andrews, R. C. (2014). Sedentary time and markers of inflammation in people with newly diagnosed type 2 diabetes. Nutrition, Metabolism and Cardiovascular Diseases, 24(9), 956962. https://doi.org/10.1016/j.numecd.2014.03.009

Fernández-Ballart, J. D., Piñol, J. L., Zazpe, I., Corella, D., Carrasco, P., Toledo, E., ... Martn-Moreno, J. M. (2010). Relative validity of a semi-quantitative food-frequency questionnaire in an elderly Mediterranean population of Spain. British Journal of Nutrition, 103(12), 1808-1816. https://doi.org/10.1017/S0007114509993837

Fröberg, A., Raustorp, A., Pagels, P., Larsson, C., \& Boldemann, C. (2017). Levels of physical activity during physical education lessons in Sweden. Acta Paediatrica, 106(1), 135-141. https://doi.org/10.1111/apa.13551

Galmés-Panadés, A., Konieczna, J., Colom, A., Morey, M., \& Romaguera, D. (2018). OBESIDAD SARCOPÉNICA Y DISFUNCIONALIDADENPOBLACIÓNCONSÍNDROME METABÓLICO. Rev.Ib.CC. Act. Fís. Dep., 8(2), 8595.

Galmes-Panades, A. M., Konieczna, J., Abete, I., Colom, A., Rosique-Esteban, N., Zulet, M. A., ... Romaguera, D. (2019). Lifestyle factors and visceral adipose tissue: Results from the PREDIMED-PLUS study. PLOS ONE, 14(1), 1-15. https://doi.org/10.1371/journal.pone.0210726

García, G. C., \& Secchi, J. D. (2014). Test course navette de 20metros con etapas de un minuto. Una idea original que perdura hace 30 años. Apunts Medicina de l'Esport, 49(183), 93-103. https://doi.org/10.1016/ j.apunts.2014.06.001

Gómez-Ruiz, M. A., \& Rodríguez Gómez, Gregorio; Ibarra Sáiz, S. (2013). COMPES: Autoinforme sobre las competencias básicas relacionadas con la evaluación de los estudiantes universitarios. Estudios Sobre Educacion, 24(24), 197-224. https://doi.org/10.15581/004.24.197-224

Hallal, P. C., Andersen, L. B., Bull, F. C., Guthold, R., Haskell, W., Ekelund, U., ... Wells, J. C. (2012). Global physical activity levels: Surveillance progress, pitfalls, and prospects. The Lancet, 380(9838), 247-257. https:// doi.org/10.1016/S0140-6736(12)60646-1

Hay, J., Maximova, K., Durksen, A., Carson, V., Rinaldi, R. L., Torrance, B., ... McGavock, J. (2012). Physical activity intensity and cardiometabolic risk in youth. Archives of Pediatrics and Adolescent Medicine, 166(11), 10221029. https://doi.org/10.1001/archpediatrics.2012.1028

Healy, G. N., Dunstan, D. W., Salamon, J., Cerin, E., Shaw, J., Zimmit, P., \& Owen, N. (2007). Objectively Measured Light-Intensity Associated With 2-h Plasma Glucose.
Diabetes Care, 30, 1384-1389. https://doi.org/10.2337/ dc07-0114.Abbreviations

Healy, G. N., Matthews, C. E., Dunstan, D. W., Winkler, E. A. H., \& Owen, N. (2011). Sedentary time and cardiometabolic biomarkers in US adults: NHANES 200306. European Heart Journal, 32(5), 590-597. https://doi.org/ 10.1093/eurheartj/ehq451

Healy, G. N., Wijndaele, K., Dunstan, D. W., Shaw, J., Salmon, J., Zimmet, P., \& Owen, N. (2008). Objectively measured sedentary time, physical activity, and metabolic risk. Diabetes Care, 31(2), 369-71. https://doi.org/10.2337/dc071795.Abbreviations

Heath, G. W., Parra, D. C., Sarmiento, O. L., Andersen, L. B., Owen, N., Goenka, S., ... Brownson, R. C. (2012). Evidence-based intervention in physical activity: lessons from around the world. Lancet, 380(9838), 272-81. https:/ /doi.org/10.1016/S0140-6736(12)60816-2

Hollis, J. L., Williams, A. J., Sutherland, R., Campbell, E., Nathan, N., Wolfenden, L., ... Wiggers, J. (2016). A systematic review and meta-analysis of moderate-tovigorous physical activity levels in elementary school physical education lessons. Preventive Medicine, 86, 34-54. https://doi.org/10.1016/j.ypmed.2015.11.018

Hu, F. B., Li, M. Y., Colditz, G. a, Willett, W. C., \& Manson, J. E. (2003). Television Watching and Other Sedentary Behaviors in Relation to Risk of Obesity and Type 2 Diabetes Mellitus in Women. Jama, 289(14), 1785. https:// doi.org/10.1001/jama.289.14.1785

Logan, G. R. M., Harris, N., Duncan, S., \& Schofield, G. (2014). A review of adolescent high-intensity interval training. Sports Medicine, 44(8), 1071-1085. https://doi.org/ 10.1007/s40279-014-0187-5

Marie Ng, Tom Fleming, Margaret Robinson, Blake Thomson, Nicholas Graetz, Christopher Margono, Erin C Mullany, S. B., Cristiana Abbafati, Semaw Ferede Abera, Jerry P Abraham, Niveen M E Abu-Rmeileh, Tom Achoki, Fadia S AlBuhairan, Z. A. A., Rafael Alfonso, Mohammed K Ali, Raghib Ali, Nelson Alvis Guzman, Walid Ammar, Palwasha Anwari, A. B., Simon Barquera, Sanjay Basu, Derrick A Bennett, Zulfi qar Bhutta, Jed Blore, Norberto Cabral, Ismael Campos Nonato, J.-C. C., Rajiv Chowdhury, Karen J Courville, Michael H Criqui, David K Cundiff, Kaustubh C Dabhadkar, Lalit Dandona, A. D., Anand Dayama, Samath D Dharmaratne, Eric L Ding, Adnan M Durrani, Alireza Esteghamati, Farshad Farzadfar, D. F. J. F., ... Jihyun Yoon, Seok-Jun Yoon, Yong Zhao, Maigeng Zhou, Shankuan Zhu, Alan D Lopez†, Christopher J L Murray†, E. G. (2014). Global, regional, and national prevalence of overweight and obesity in children and adults during 1980-2013: a systematic analysis for the Global Burden of Disease Study 2013. The Lancet, 384(9945), 766-781. https://doi.org/10.1016/S01406736(14)60460-8

Martínez-González, M. A., López-Fontana, C., Varo, J. J., Sánchez-Villegas, A., \& Martinez, J.A. (2005). Validation of the Spanish version of the physical activity questionnaire used in the Nurses' Health Study and the Health Professionals' Follow-up Study. Public Health Nutrition, 8(07), 920-927. https://doi.org/10.1079/ PHN2005745 
Matthews, C. E., Chen, K. Y., Freedson, P. S., Buchowski, M. S., Beech, M., Pate, R. R., \& Troiano, R. P. (2008). Amount of Time Spent in Sedentary Behaviors in the United States, 2003-2004, 167(7), 875-881. https://doi.org/ 10.1093/aje/kwm390.Amount

Monleón C., Fargueta, M. y Elvira, L. (2015). PIAFARC EFFECTS ON DENSITOMETRY IN OBESE PHYSICAL CONDITION. RICCAFD, 4(3), 11-20.

Muntaner-Mas, A., Vidal-Conti, J., Cantallops, J., Borràs, P. A., \& Palou, P. (2017). Obesity and physical activity patterns among Balearic Islands children and adolescents: A cross-sectional study. Journal of Human Sport and Exercise, 12(2), 333-348. https://doi.org/10.14198/ jhse.2017.122.10

Organización mundial de la salud(OMS). (2005). Instrumento del método progresivo ( STEPS ). Organización Mundial de La Salud 20 Avenue Appia, 1211 Ginebra 27, Suiza, 17. https://doi.org/10.1016/j.seares.2018.02.007

Organización mundial de la salud (OMS). (2012). Global Physical Activity Questionnaire (GPAQ) Analysis Guide. Surveillance and Population-Based Prevention Prevention of Noncommunicable Diseases Department World Health Organization 20 Avenue Appia, 1211 Geneva 27, Switzerland For Further Information: Www.Who.Int/Chp/Steps, 1-22. https://doi.org/10.1016/ S0140-6736(12)60736-3.The

Padilla, J. (2014). Relación Del Índice De Masa Corporal Y El Porcentaje De Grasa Corporal En Jóvenes Venezolanos Relationship of Body Mass Index and Body Fat Percentage in Young Venezolanos. Rev.Ib.CC. Act. Fis. Dep, 3(1), 27-33.

Peters, J. C., Beck, J., Cardel, M., Wyatt, H. R., Foster, G. D., Pan, Z., ... Hill, J. O. (2016). The effects of water and nonnutritive sweetened beverages on weight loss and weight maintenance: A randomized clinical trial. Obesity, 24(2), 297-304. https://doi.org/10.1002/oby.21327

Piercy, K. L., Troiano, R. P., Ballard, R. M., Carlson, S. A., Fulton, J. E., Galuska, D. A., ... Olson, R. D. (2018). The Physical Activity Guidelines for Americans. Jama, 9762, 1-9. https://doi.org/10.1001/jama.2018.14854

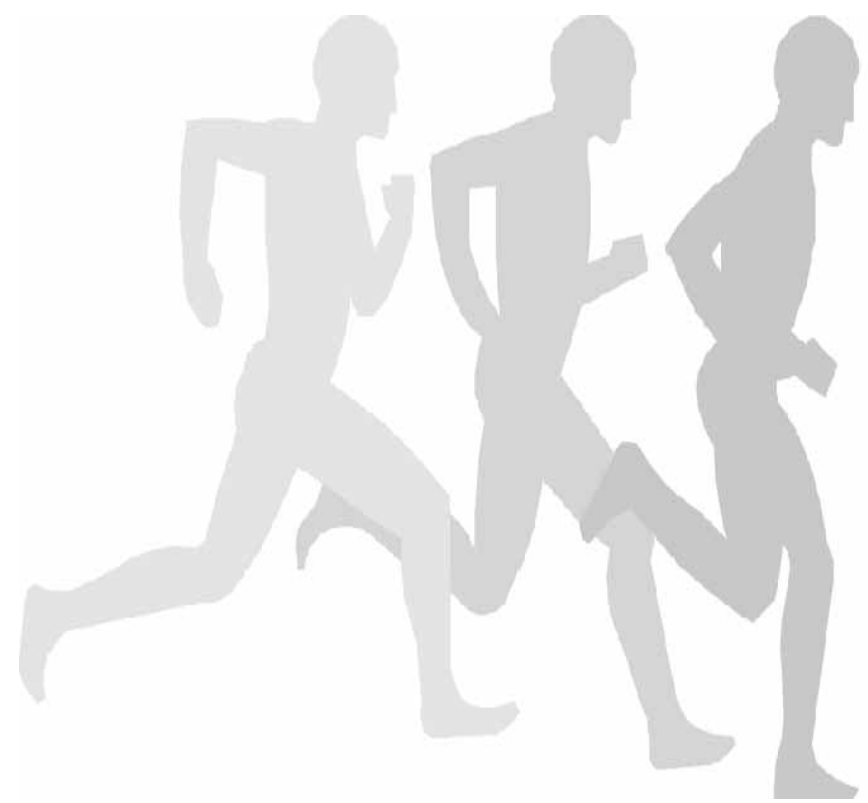

Rodríguez-Gómez, G., Ibarra-Sáiz, M. S., \& Gómez-Ruiz, M. Á. (2010). e -Autoevaluación en la universidad/ : un reto para profesores y estudiantes e -Self-Assessment in Higher Education/ : a Challenge for Teachers and Students. Revista de Educación, 401-430. https://doi.org/ 10-4438/1988-592X-RE-2010-356-045

Rodríguez Gómez, Gregorio; Ibarra Saiz, María Soledad; Cubero Ibáñez, J. (2018). COMPETENCIAS BÁSICAS RELACIONADAS CONLAEVALUACIÓN. UNESTUDIO SOBRELAPERCEPCIÓNDELOSESTUDIANTESUNIVERSITARIOS (BASIC. South African Journal of Geology, 21(2), 181-208. https://doi.org/10.5944/ educXX1.14457

Stenholm, S., Rantanen, T., Heliövaara, M., \& Koskinen, S. (2008). The mediating role of C-reactive protein and handgrip strength between obesity and walking limitation. Journal of the American Geriatrics Society, 56(3), 462469. https://doi.org/10.1111/j.1532-5415.2007.01567.x

Tremblay, M. (2012). Letter to the editor: standardized use of the terms \&quot;sedentary\&quot; and \&quot;sedentary behaviours\&quot;. Applied Physiology, Nutrition, and Metabolism, 37(3), 540-2. https://doi.org/10.1139/h2012024

Vidal-conti, J. (2016). el modelo socio-ecológico mediante un análisis multifactorial to a socio-ecological model using multifactorial analysis, 51-59.

Villardón-Gallego, M. L. (2006). Evaluación del aprendizaje para promover el desarrollo de competencias. Educatio Siglo XXI, 24, 57-76. https://doi.org/http:// d i a l n e t. u n i ri o j a . e s/s e r v l e t/ dcart?info=link\&codigo=2339652\&orden=128237

Wilmot, E. G., Edwardson, C. L., Achana, F. A., Davies, M. J., Gorely, T., Gray, L. J., .. . Biddle, S. J. H. (2012). Sedentary time in adults and the association with diabetes, cardiovascular disease and death: systematic review and meta-analysis. Diabetologia, 55(11), 2895-2905. https:/ /doi.org/10.1007/s00125-012-2677-z

World Health Organization. (2010). World Recommendations on Physical Activity for Health. Geneva: WHO Library Cataloguing-in-Publication, (Completo), 1-58. https:// doi.org/978924359997 7

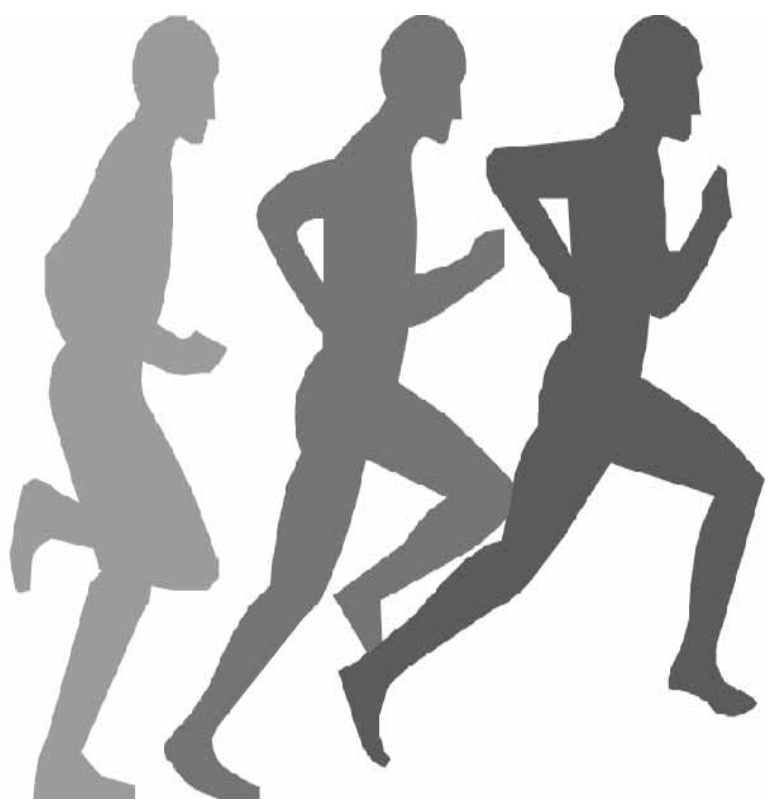

